JOURNAL OF SYNCHROTRON RADIATION

ISSN 1600-5775

Received 2 March 2021

Accepted 29 April 2021

Edited by R. W. Strange, University of Essex, United Kingdom

₹ These authors contributed equally to this work and are both joint first authors.

Keywords: X-ray; ptychography; nuclei; quantitative phase imaging; synchrotron.

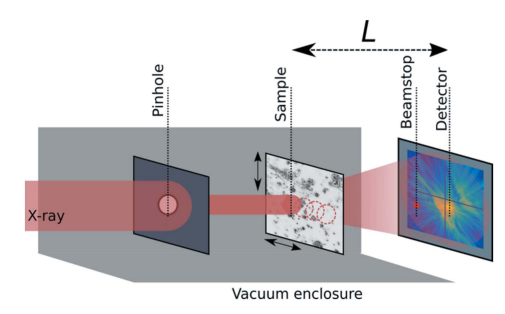

\section{Quantitative phase measurements of human cell nuclei using X-ray ptychography}

\author{
Jorg Schwenke, ${ }^{a, b, c *} \neq$ Mohammed Yusuf, ${ }^{a, b, d} * \neq$ Laura A. Shemilt, ${ }^{a, b}$ \\ Ulrich Wagner, ${ }^{e}$ Atiqa Sajid, ${ }^{d}$ Graeme R. Morrison, ${ }^{a, b}$ Fucai Zhang, ${ }^{a, b}$ \\ Aaron Parsons, ${ }^{e}$ Christoph $\operatorname{Rau}^{\mathrm{e}}$ and Ian K. Robinson ${ }^{\mathrm{a}, \mathrm{b}, \mathrm{f}}$
}

\footnotetext{
aLondon Centre for Nanotechnology, University College London, 17-19 Gordon Street, London WC1H 0AH, United Kingdom, ${ }^{\mathbf{b}}$ Research Complex at Harwell (RCaH), Rutherford Appleton Laboratory, Harwell, Oxford OX11 OFA, United Kingdom, 'MAX IV Laboratory, Lund University, PO Box 118, SE-22100 Lund, Sweden, ${ }^{\mathbf{d}}$ Centre for Regenerative Medicine and Stem Cell Research, Aga Khan University, Karachi, Pakistan, e Diamond Light Source Ltd, Diamond House, Harwell Science and Innovation Campus, Didcot OX11 ODE, United Kingdom, and ${ }^{\mathfrak{f} C}$ Condensed Matter Physics and Materials Department, Brookhaven National Laboratory, Upton, NY 11973, USA. *Correspondence e-mail: jorg.schwenke@maxiv.lu.se, ucanymo@ucl.ac.uk
}

The human cell nucleus serves as an important organelle holding the genetic blueprint for life. In this work, X-ray ptychography was applied to assess the masses of human cell nuclei using its unique phase shift information. Measurements were carried out at the I13-1 beamline at the Diamond Light Source that has extremely large transverse coherence properties. The ptychographic diffractive imaging approach allowed imaging of large structures that gave quantitative measurements of the phase shift in $2 \mathrm{D}$ projections. In this paper a modified ptychography algorithm that improves the quality of the reconstruction for weak scattering samples is presented. The application of this approach to calculate the mass of several human nuclei is also demonstrated.

\section{Introduction}

The cell nucleus is surrounded by the nuclear envelope and separates the chromosomes from the cytoplasm. It is the storage place for genetic information contained in DNA (Pederson, 2011; Alberts et al., 2014). It is also here that important processes of gene expression, replication, recombination and repair, RNA processing and ribosome sub-unit assembly occur, making the nucleus a defining feature of eukaryotic cells as it determines cell fate (Rippe, 2007; Lamond \& Earnshaw, 1998). Alterations of nuclear physical properties have been linked with various diseases (Dahl et al., 2008). In human cells, the nucleus averages $10 \mu \mathrm{m}$ in diameter in its natural state (Alberts et al., 2014). Its size increases over time until it reaches late prophase (Webster et al., 2009). The nucleus shell is formed by a porous membrane which is filled with nucleoplasm and contains the chromatin (protein-DNA complex) and various other sub-nuclear bodies at certain stages of the cell cycle (Zidovska, 2020). Even though the human nucleus has been studied in great detail, the 'rich inner life' is still under investigation (Zidovska, 2020).

Phase-contrast microscopy, that provides quantitative information, can be promising for assessing clinical samples (Wang et al., 2010). In this work, we use coherent X-ray diffraction to image human cell nuclei and extract quantitative information relating to the mass. X-ray diffraction can yield high spatial resolution images in 2D and is suitable for tomography schemes as well (Nishino et al., 2009; Dierolf et al., $2010 a$ ). The thickness of the sample requires the use of hard $\mathrm{X}$-rays, with a photon energy above $6 \mathrm{keV}$ to provide the 
necessary penetration depth. However, due to the reduced absorption, the diffraction efficiency mostly relies on the phase change due to the refractive index in the sample. This makes samples with low electron density (such as biological samples) notoriously difficult to image with hard X-rays, because parasitic scattering from beamline components (e.g. apertures, vacuum windows) can become dominant and appears prominently in the acquired diffraction patterns. These contributions can be greatly reduced by careful beamline design. They can also be eliminated by employing imaging methods that factor out the illumination conditions, such as $\mathrm{X}$-ray ptychography, which we use here.

In ptychography, the sample is scanned in steps smaller than the illumination size and a large number of diffraction patterns is collected (Faulkner \& Rodenburg, 2004; Thibault et al., 2008). Each individual diffraction pattern can be seen as a 'view' of the sample, corresponding to a specific area in the two-dimensional sample plan, and containing the diffraction information from this area. It is represented by at least two arrays in the sample plane, one (or more) representing the illumination function, and one (or more) representing the sample view. Because the views overlap, they contain redundant information and, by assuming that the illumination is constant (or varying slowly), the diffraction contribution from the sample can be isolated and a complex real-space image of the sample can be reconstructed through iterative phase retrieval. In practice, numerical phase retrieval can still be difficult when the diffracted intensity is close to the noise level, as it is with the samples in this work. Standard implementations of the common numerical algorithms do not yield satisfactory convergence in every case, but stagnate without producing a unique solution that is in agreement with the diffraction data (Dierolf et al., 2010b). Over the past years, many improvements to the algorithms have been suggested that improve the convergence of the algorithm or use constraints to remove artefacts (e.g. Thibault \& GuizarSicairos, 2012; Huang et al., 2014; Maiden et al., 2017). We have found that one particular refinement of the algorithm, reciprocal space sub-sampling, first suggested by Batey et al. (2014), leads to significant improvement in the phase retrieval of weakly scattering samples.

In this article, we describe the implementation of a refined ptychography algorithm and its application to human nuclei. We also discuss the use of the retrieved phase information to quantify the mass distribution of the sample, based on assumptions of the molecular composition.

\section{Material and methods}

\subsection{Cell culture and nuclei preparation}

Cells were grown according to previously published protocols (Yusuf et al., 2014a,b). A B-lymphocyte male Yoruba cell line (passage 4) (GM18507) was cultured at $37^{\circ} \mathrm{C}$ in a $5 \%$ $\mathrm{CO}_{2}$ atmosphere using RPMI medium supplemented with $100 \mathrm{U} \mathrm{ml}^{-1}$ penicillin and $100 \mu \mathrm{g} \mathrm{ml}^{-1}$ streptomycin (Gibco), $20 \%$ FBS (reagents purchased from Sigma-Aldrich, Dorset,
UK) and 1\% L-glutamine (Sigma Aldrich, UK). Cells were arrested in metaphase after treatment with Colcemid $\left(0.2 \mu \mathrm{g} \mathrm{ml}^{-1}\right.$, Gibco BRL) $\left[0.2 \mu \mathrm{g} \mathrm{ml}^{-1}\right.$ (Gibco Life Technologies, Paisley, UK)] overnight followed by KCL $(0.075 \mathrm{M})$ (VWR BDH Prolabo, Dublin, Ireland) treatment at $37^{\circ} \mathrm{C}$ for 5 min. Nuclei were extracted and fixed in Carnoy's solution (3:1 methanol:acetic acid) and $0.5 \%$ glutaraldehyde (Sigma) (10 $\mathrm{m} M$ Hepes- $\mathrm{KOH}$ and $5 \mathrm{mM} \mathrm{MgCl}$ ) according to Shemilt et al. (2015). The sample material was placed onto $100 \mathrm{~nm}$ thick silicon nitride windows (Silson, UK) and stained with $150 \mu M$ Syber gold dye. The sample was washed using water and then left to air dry. The samples were imaged using a Zeiss AxioZ2 microscope with Isis software (Metasystems) to provide both visible and fluorescence images for reference and correlation. Overall, three samples, numbered 1, 2 and 3, were used in the study; samples 1 and 2 were stained with platinum blue (Pt) (Wanner \& Formanek, 1995; Yusuf et al., 2014a) at $5 \mathrm{~m} M$ concentration for 30 minutes and washed in water.

\subsection{X-ray ptychography data acquisition}

The experiments were carried out at the I13-1 coherence beamline at the Diamond Light Source in Harwell, UK (Pešić et al., 2013). For this experiment a photon energy of $7.5 \mathrm{keV}$ was used and the $\mathrm{X}$-ray beam was collimated by a compound refractive lens at the front-end. I13-1 is specifically designed to maximize transverse coherence: it consists of a $250 \mathrm{~m}$ section between the front-end and the monochromator which allows for a transverse coherence length exceeding $200 \mu \mathrm{m}$ in the vertical direction (Rau et al., 2011). After the monochromator, the beam was cropped by a pinhole aperture of diameter $D=$ $20 \mu \mathrm{m}$, so that the X-ray probe on the sample plane is larger than the expected size of a human nucleus. The sample was mounted on a stack of piezo-electric translation stages in a vacuum environment at a pressure of about 10 mbar during the measurements, without active pumping. There were no vacuum windows between the pinhole aperture and the far end of the flight tube, where a Kapton window was installed directly in front of the detector, a MEDIpix2 (de Vries et al., 2007), a discrete pixel-array detector with pixel size $p=55 \mu \mathrm{m}$. The distance between the sample and the detector was about

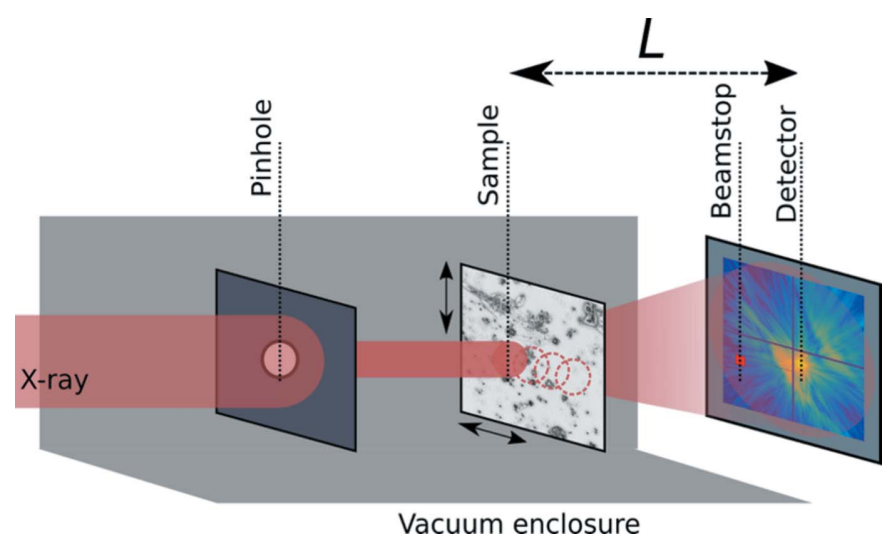

Figure 1

Experimental imaging setup at Diamond Light Source I13-1. 


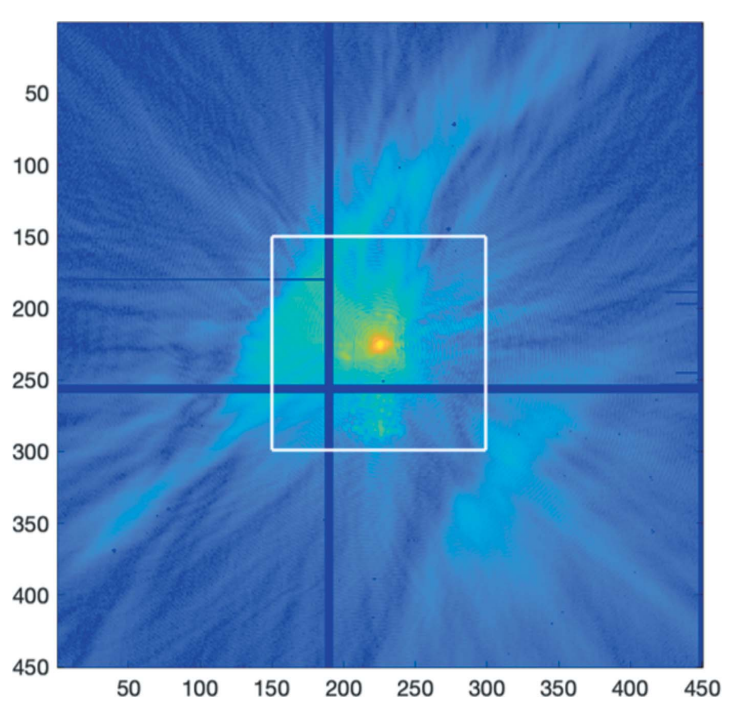

Figure 2

Full detector frame from a nuclei dataset (log plot). The zero order is centred. The dark blue lines represent missing data due to gaps between the detector areas and dead pixels. The white square marks the cropped area shown in Fig. 6.

$L=15 \mathrm{~m}$, and the array size is cropped to $450 \times 450$ pixels, which gives a corresponding real-space pixel size of $200 \mathrm{~nm}$ at $7.5 \mathrm{keV}$. A partially transparent beamstop, made of tungsten with a thickness of $10 \mu \mathrm{m}$, was glued to a separate Kapton foil and placed in front of the detector to attenuate the zero-order beam. A schematic diagram of the imaging setup is shown in Fig. 1. Fig. 2 shows a typical diffraction pattern acquired from the measurements. Typical scan parameters were $5 \mathrm{~s}$ exposure time per diffraction pattern, and average step size of $2 \mu \mathrm{m}$ between scan positions, which were distributed to fill a circle using Poisson disk sampling (Dunbar \& Humphreys, 2006) and sorted into a spiral scan path trajectory.

\subsection{Phase retrieval algorithm}

Ptychography is an iterative phase retrieval scheme that aims to find the correct complex phase for each pixel of a set of measured diffraction patterns, where only the intensities are recorded. A full description of the algorithm can be found in Maiden \& Rodenburg (2009). Here we will focus on the sampling aspect and the reciprocal-space sampling, which is based on the physical pixel size of the detector and the experimental geometry. The recorded intensities in the reciprocal plane are the square modulus of the far-field diffraction patterns. The recorded intensity $I_{j}(\mathbf{u})$ at reciprocal-space coordinate $\mathbf{u}$ of diffraction pattern $j$ can be calculated from the probe function $P$ and the object function $O$ as

$$
I_{j}(\mathbf{u})=\left|\mathcal{F}\left[O(\mathbf{r}) P\left(\mathbf{r}-\mathbf{R}_{j}\right)\right]\right|^{2},
$$

where $\mathcal{F}$ is the two-dimensional Fourier transform, $\mathbf{r}$ is a coordinate vector in sample space and $\mathbf{R}_{j}$ is the movement of the probe relative to the object for the $j$ th measurement. The product $O(\mathbf{r}) P\left(\mathbf{r}-\mathbf{R}_{j}\right)$ forms the exit wave $\psi_{j}(\mathbf{r})$ for that particular view.
According to Edo et al. (2013), the experimental geometry must be set such that the smallest interference fringes can be sampled by the detector,

$$
\frac{L \lambda}{D p}>2 .
$$

The linear oversampling ratio $S_{\mathrm{o}}$ describes by how much the minimum requirement is exceeded in any given experimental geometry,

$$
S_{\mathrm{o}}=\frac{L \lambda}{2 p} \frac{1}{D} .
$$

Values of $S_{\mathrm{o}}$ larger than 1 are theoretically sufficient for phase retrieval but often require a priori knowledge of either the illumination or the sample. It has been shown recently (Edo et al., 2013) that ptychography can be used even in undersampled conditions, $S_{\mathrm{o}}<1$, as the overlap redundancy in real space compensates for the under-sampling in diffraction space. Edo et al. showed that the step size by which the illumination is shifted between exposures is a crucial parameter in ptychography, and they provide a useful figure of merit that we will refer to as the ptychography sampling ratio $S_{\mathrm{p}}$, where

$$
S_{\mathrm{p}}=\frac{L \lambda}{2 p} \frac{1}{R}
$$

Here, $R$ denotes the average step-size in between exposures. This measure replaces the pinhole size $D$ from the linear oversampling ratio, as the aperture size is irrelevant in ptychography. In this formulation, sampling ratios $S_{\mathrm{p}}$ larger than 1 are considered sufficient for successful reconstruction. The step-size $R$ can be adjusted to compensate for either a large pixel size $p$ or a short detector distance $L$, thus allowing for geometries with large detector acceptance angle capable of producing reconstructions with high spatial resolution.

In our experimental geometry, the linear oversampling criterion was just met: $S_{\mathrm{o}}=1.2$. This is due to the large diameter of the pinhole, which was chosen to exploit the excellent transverse coherence of the beam and increase the total X-ray flux. However, our setup reaches values of $S_{\mathrm{p}}$ between 10 and 30, depending on the scan pattern used. Under these conditions, we found that ptychography scans of strongly scattering samples, such as a test sample made of tungsten, could be reconstructed without any a priori knowledge of the illumination function.

We here employ a reciprocal-space up-sampling algorithm as suggested by Batey et al. (2014), in which the pixels in the diffraction patterns are split into sub-pixels to simulate a detector with smaller physical detector pitch than actually used. The method can be used to reconstruct diffraction data that nominally violates the ptychography sampling condition, but they also report a general improvement in the quality of the reconstruction. They note that, even in cases that fulfil the sampling condition, the quality of the reconstruction can be improved. We use this method to obtain good reconstructions of our weakly scattering data.

We use $N$ to denote the number of pixels that take up one physical space along each dimension, so that each pixel is 
replaced by $N^{2}$ sub-pixels. Due to the properties of the Fourier transform, a finer pixel pitch in reciprocal space results in a larger field of view in real space. For the iterative phase retrieval, the real-space arrays for the illumination function and the initial guess for each view are therefore padded with zeros on all sides to increase the array size by a factor of $N^{2}$. The exit wave is propagated by Fourier transformation to the detector plane, where each physical detector pixel $m$ is now represented by $N^{2}$ virtual pixels labelled $n$. In the detector plane the modulus constraint is applied by comparing the measured pixel intensity $I_{m}$ with the virtual sub-pixels. Each artificial pixel $\Psi_{j, m, n}^{\prime}$ is updated according to

$$
\Psi_{j, m, n}^{\prime}=\Psi_{j, m, n} \frac{\sqrt{I_{m}}}{\left(\sum_{n=1}^{N^{2}}|\Psi|_{j, m, n}^{2}\right)^{1 / 2}} .
$$

In simple terms, the squared sum of the artificial sub-pixels is made to agree with the measured intensity $I_{m}$ for the corresponding real pixel, by a normalization coefficient, and multiplying the coefficient with each sub-pixel, thus preserving the ratio between sub-pixels values. The modified function $\Psi_{j, m, n}^{\prime}$ is then propagated back to the object plane by inverse Fourier transform to determine the updated exit wave $\Psi_{j}^{\prime}(\mathbf{r})$ in the object plane. As was seen before, the use of sub-sampling results in an expanded object function in the real space. However, the data in this region are unconstrained and deteriorate into numerical noise.

\subsection{Quantitative analysis}

The phase shift $\phi$ is reconstructed quantitatively in units of the wavenumber of the illumination as a projection in the direction of X-ray propagation. The quantitative phase shift can be directly converted into a spatial map of the projected electron density, $\sigma_{\mathrm{e}}$, based on the classical electron radius $r_{0}$ (Giewekemeyer et al., 2010),

$$
\sigma_{\mathrm{e}}=-\frac{\phi}{\lambda r_{0}} .
$$

The electron density can be therefore used to estimate the mass thickness of the imaged features using an averaged atomic mass number $A$, an average atomic number $Z$, and the atomic mass unit $u$,

$$
\sigma_{m}=\frac{A u}{Z} \sigma_{\mathrm{e}}
$$

For human cell nuclei, which mostly consist of light elements $(\mathrm{C}, \mathrm{H}, \mathrm{N}, \mathrm{O})$, the ratio $A / Z$ is very close to 2 . We can integrate the mass density of nuclei identified in the reconstructions and estimate the total mass.

\section{Results}

The samples containing cell nuclei are composed of mostly low- $Z$ elements, which are almost transparent to hard $\mathrm{X}$-rays and only cause small phase changes in the illumination wavefront. The diffraction patterns collected from these samples are consequently dominated by diffraction at the

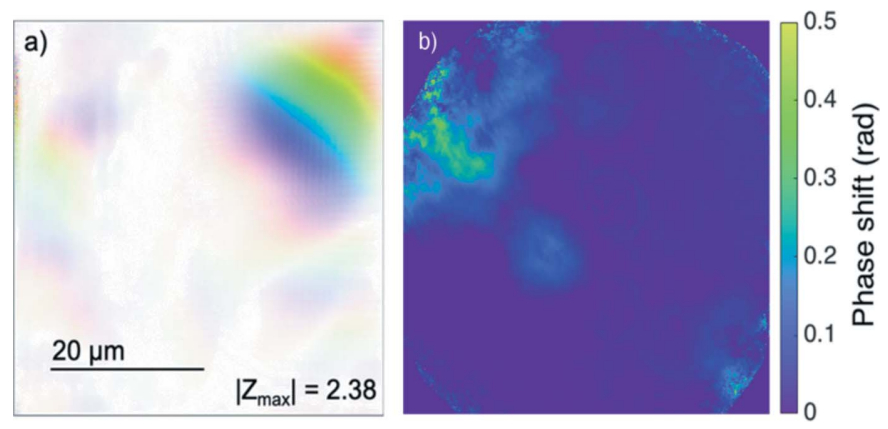

Figure 3

Reconstruction of a dataset using actual pixel size. The probe function [ $(a)$, HSV plot, see Fig. 4(a) for legend] is not recovered properly, but is off-centre and distributed over the entire array. The reconstructed phase $[(b)$, phase plot $]$ is blurry.

pinhole aperture that was used to define the beam, as shown in Fig. 2.

A clear image of the sample could not be reconstructed from these datasets, even with detailed knowledge of the illumination obtained from reconstructions of the test samples. Fig. 3 shows a reconstruction made using a standard algorithm described by Maiden \& Rodenburg (2009), while Fig. 4 shows a reconstruction made with the modified algorithm using sub-pixels $(2 \times 2)$. The figures show both the reconstructed complex probe array as an HSV plot, and the reconstructed phase shift of the object. The reconstruction using sub-pixels is significantly better in both parts; the probe function in particular is a far better representation of the pinhole. The sample function is also more detailed and less blurry.

One way to assess the performance of the phase retrieval is the use of an error function in the reciprocal plane, where the current iterate is compared with the measured data,

$$
\operatorname{Err}=\sum_{j} \frac{\sum_{m}\left|\sqrt{I_{m}}-\right| \Psi_{m}||^{2}}{\sum_{m}\left|\sqrt{I_{m}}\right|^{2}} .
$$

Here, $I_{m}$ is the intensity measured in pixel $m$ of diffraction pattern $j$, while $\Psi_{m}$ is the retrieved complex value for the same pixel. The virtual sub-pixels are summed before the error is calculated. Fig. 5 shows the error functions for the two reconstructions in Figs. 3 and 4. The error can be reduced by

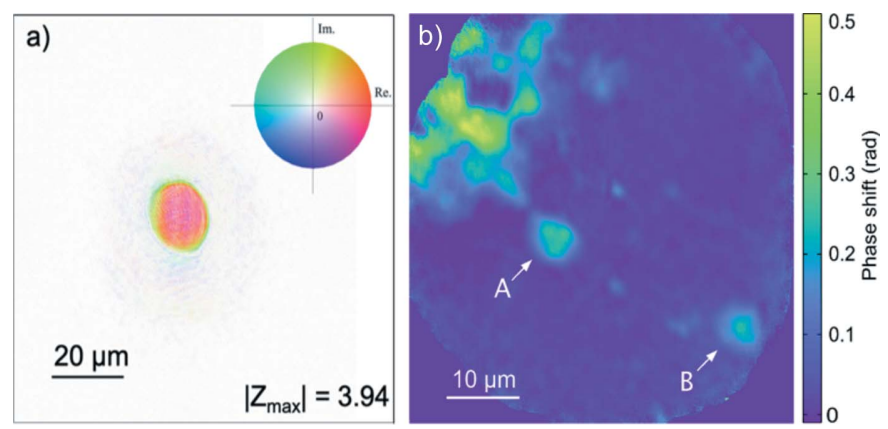

Figure 4

Reconstruction using virtual sub-pixels $(2 \times 2),(a)$ probe function $(\mathrm{HSV}$ plot) and (b) reconstructed phase shift. 


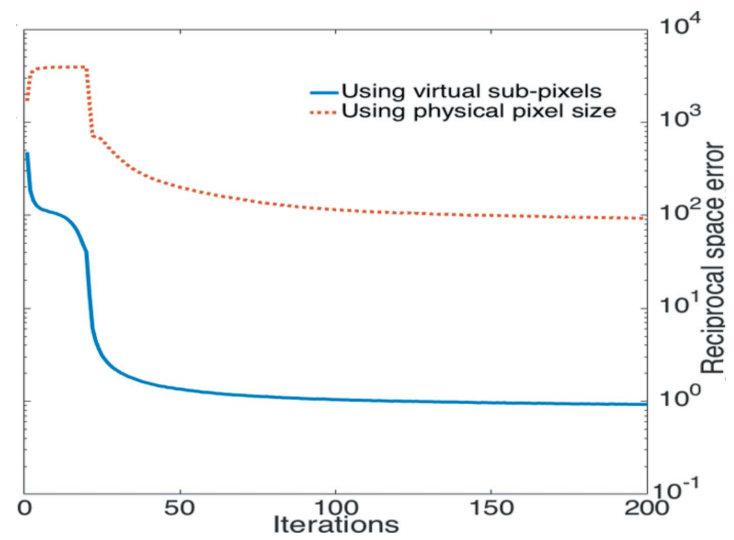

Figure 5

Comparison of the error function for phase retrieval with physical pixel size and with virtual sub-pixels $(2 \times 2)$.
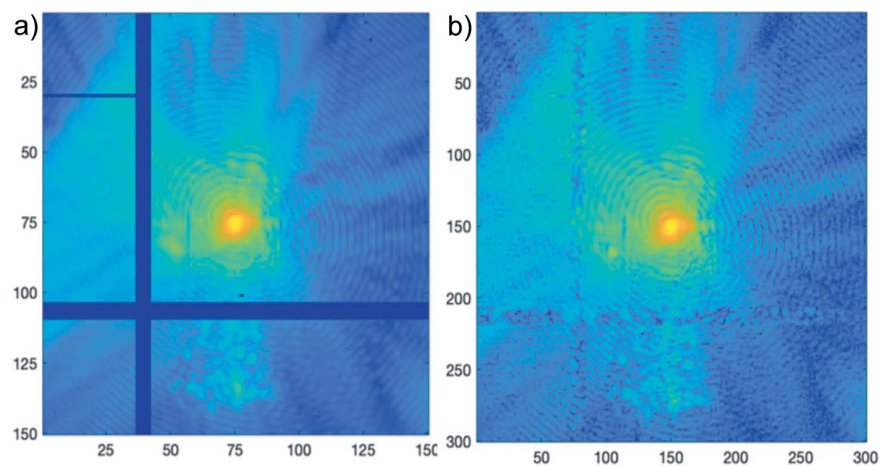

Figure 6

Comparison between the measured diffraction pattern $[(a), \log$ plot $]$ and the up-sampled iterated exit wave in the detector plane $[(b), \log$ plot]. The numbers represent pixels.

almost two orders of magnitude by sub-sampling the data during the reconstruction process. Fig. 6 shows a comparison between the measured data $(a)$ and the final iterate $(b)$, the exit wave corresponding to the same diffraction pattern as in Figs. 2 and $6(a)$. The area shown is cropped from the full array, illustrated by the white rectangle in Fig. 2. As can be seen, the pixel size is smaller in Fig. 6(b), due to the use of sub-pixels. The algorithm has filled in the missing data from the measurement with data from other diffraction patterns.

Fig. 7 shows the Fourier ring correlation function (FRC) for the stained nucleus sample 1 shown in Figs. 3 and 4. The FRC indicates the information validity of the reconstruction by comparing two reconstructions of the same object. The abscissa shows full spatial period of the frequency components in the diffraction data. The data cut off at about $400 \mathrm{~nm}$ (200 $\mathrm{nm}$ half-period) due to the size of the detector array (see above). The FRC function is compared with the $3 \sigma$ threshold (van Heel \& Schatz, 2005), and is above the threshold and therefore valid over the spatial frequency interval observed. Based on the sampling conditions and the visible features, we find the resolution to be $200 \mathrm{~nm}$, the physical pixel size in the sample plane, and one half-period of the largest diffracted frequency measured by the detector.

A total of 13 datasets were used for the quantitative analysis. For each dataset, the phase retrieval was repeated

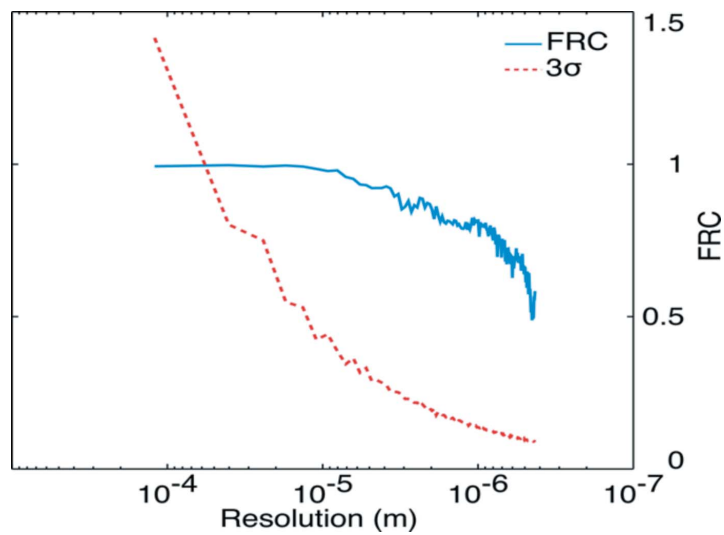

Figure 7

Fourier ring correlation of two separate and independent measurements of the same field of view. The red line shows the $3 \sigma$ noise level (van Heel \& Schatz, 2005).

30 times with different random seeds and the reconstructed phase shift was averaged. A histogram of the pixel phase values was used to determine the phase offset for the membrane surrounding the nuclei. The nuclei were isolated from the background by setting a threshold at three standard deviations above the normal distribution peak of membrane background. In cases where nuclei were not completely isolated, watershed segmentation was applied using the MATLAB in-built function to define the nucleus. Fig. 8 shows segmented nuclei A and B as identified in Fig. 4(b).

The recovered phase properties of the nuclei were then used according to equations (6) and (7) to calculate the integrated mass. The area was determined by counting the number of pixels that each nucleus occupies above the threshold. Table 1 shows a comparison of the average mass, area and mass density for the three samples, while Fig. 9 shows a histogram of all the nuclei examined [Samples 1 (blue) and 2 (yellow) are stained with Pt and Sample 3 (red) is unstained].

Overall, a varied range of masses were observed from all the samples. Samples 1 and 2 displayed a cluster approximately between 30 and $80 \mathrm{pg}$. Sample 1 had two nuclei with a higher mass of 140 and $190 \mathrm{pg}$. Sample 3 showed a cluster approximately around $50 \mathrm{pg}$ with the two nuclei displaying a mass above $80 \mathrm{pg}$.

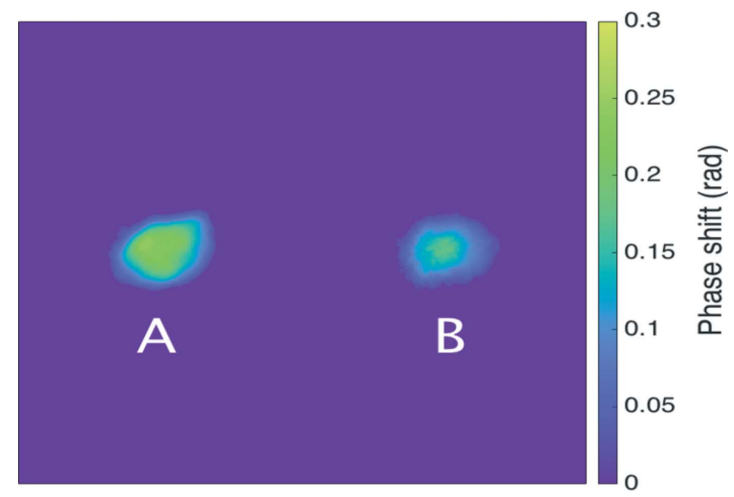

Figure 8

Isolated nuclei from the data shown in Fig. 2(a). 

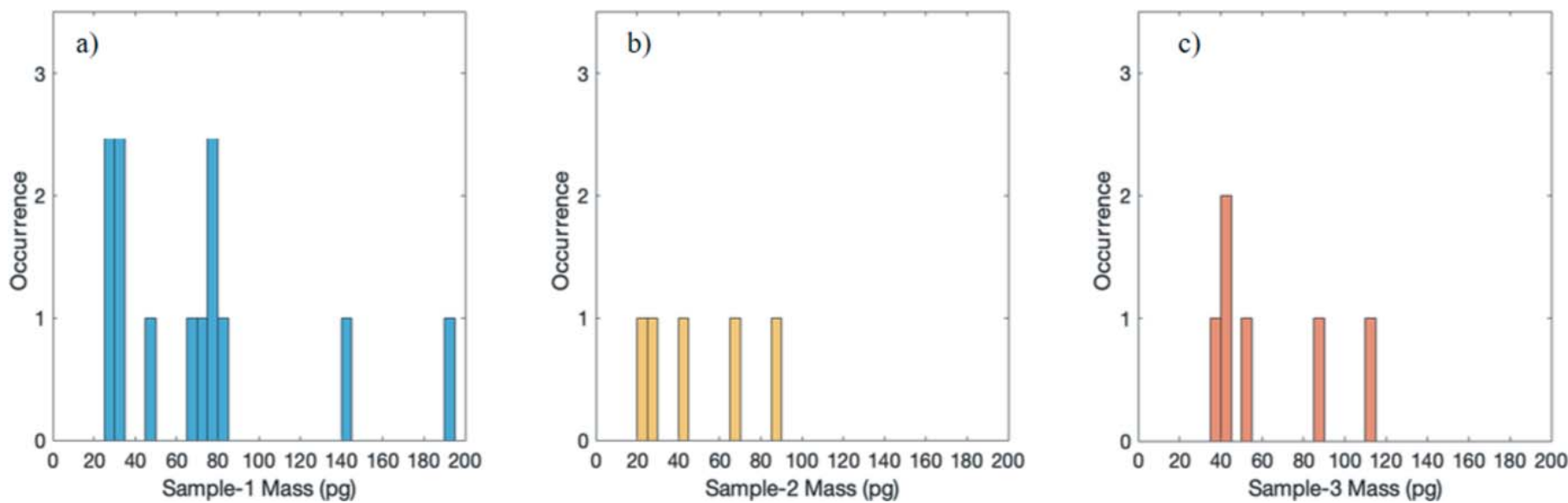

Figure 9

Histogram of observed nuclei mass. Sample 1 (stained) is shown in blue, Sample 2 (stained) is shown in yellow, and Sample 3 (unstained) is shown in red. The height of the bars indicates the number of nuclei in a specific mass interval. Note that the three samples are shown separately and not as a sum.

Table 1

Average mass, area and mass density for the three samples.

\begin{tabular}{llll}
\hline & Mass $(\mathrm{pg})$ & Area $\left(\mu \mathrm{m}^{2}\right)$ & Mass density $\left(\mathrm{pg} \mu \mathrm{m}^{-2}\right)$ \\
\hline Sample 1 & $75.1 \pm 50.6$ & $26.4 \pm 10.3$ & $2.7 \pm 1.0$ \\
Sample 2 & $91.1 \pm 90.0$ & $25.9 \pm 11.6$ & $3.0 \pm 1.7$ \\
Sample 3 & $49.7 \pm 23.7$ & $58.5 \pm 15.5$ & $0.84 \pm 0.32$ \\
\hline
\end{tabular}

Finally, Fig. 10 shows the relationship between the occupied area of the nuclei and their respective mass. A linear regression is also plotted for each sample. The plot shows a linear relationship between the area and mass. Samples 1 (blue) and 2 (yellow) are stained with Pt and Sample 3 (red) is unstained. The three samples showed differences in the occupied area for nuclei measured. For each sample an increase in mass was observed with increase in occupied area.

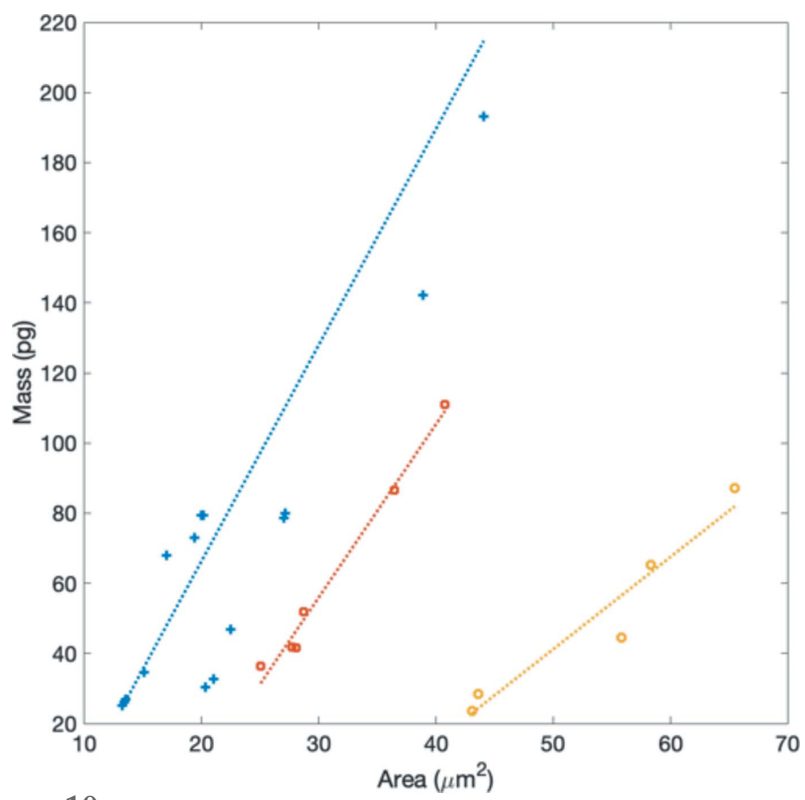

Figure 10

Observed nuclei mass against occupied area. Sample 1 (stained) is shown in blue, Sample 2 (stained) is shown in yellow, and Sample 3 (unstained) is shown in red.

\section{Discussion}

In this study, X-ray ptychography was performed on human cell nuclei giving quantitative measurements of the phase allowing a direct estimation of the mass. We find that ptychography is suitable for routine imaging of weak phase objects such as organic tissue illuminated with hard X-ray radiation. The application of reciprocal-space up-sampling during the iterative phase retrieval was crucial in the presented study and could potentially become a standard modification when dealing with weak phase materials.

As shown in Fig. 3, the error was significantly reduced in this way as the reconstructed image appears to be more detailed, as shown in Fig. 2. The method does not affect the theoretical resolution of the reconstructed image because the up-sampling in the detector plane transfers to an extended object and illumination functions in real space. However, in practice, the reconstruction matches much more closely the true values of the sample and therefore smaller features of the sample become visible.

The obtained nuclei masses of the unstained samples were around $40 \mathrm{pg}$. Compared with unstained samples, Pt-stained samples showed a varied range of masses between 30 and $80 \mathrm{pg}$. No major changes in the mass between unstained and stained samples was seen except for two nuclei that displayed a mass above $80 \mathrm{pg}$ which may be due to additional Pt residue during sample preparation. Other studies have also observed a wide range of masses after performing quantitative electron microscopy on human interphase nuclei from blood lymphocytes (Golomb \& Bahr, 1974). Overall, a bimodal distribution was seen with a mass range of $65 \mathrm{pg}$ and $88 \mathrm{pg}$ (Golomb \& Bahr, 1974) that is in close agreement with the findings of this study. Other studies have determined the mass of human nuclei ranging in the lower end 45-55 pg (Sandritter et al., 1963; Sandritter \& Müller, 1959) and higher end 71.9383.59 pg (Bahr \& Golomb, 1971).

A possible reason for variation seen in our study is that the sample might have been at different stages of the cell cycle. As the nucleus resides inside the cell throughout interphase $\left(\mathrm{G}_{1}\right.$, 
$\mathrm{S}$ and $\mathrm{G}_{2}$ ), it reaches prophase where the nuclear envelope breaks down that is then rebuilt back at telophase. As a cell progresses through the cell cycle, the size of the nucleus monotonously increases over hours and undergoes various reorganization including its size and DNA/protein content (Chu et al., 2017). As there is a broad range of determined nucleus masses found in our data, we can divide our population sampled into two different states between 30 and $50 \mathrm{pg}$ and between 65 and $90 \mathrm{pg}$, indicating that they may be in $\mathrm{G}_{1}$ to early $S$ and late $S$ to $G_{2}-M$, respectively. This difference can be interpreted as due to cells in metaphase (after DNA replication) and interphase (before DNA replication).

The DNA content of a nucleus is diploid $(2 N)$ in $G_{1}$ and early $\mathrm{S}$ phase and is tetraploid $(4 N)$ in late $\mathrm{S}$ phase through to M (Yang, 2018). The expected DNA mass for the full haploid human genome $\left(3.5 \times 10^{9}\right.$ base pairs of DNA) is $3.5 \mathrm{pg}$ for a single strand of double helix, plus $5 \mathrm{pg}$ of protein in the known histones with one octamer per 170 base pairs (Woodcock \& Frank, 1984). Known non-histone proteins account for $50 \%$ more, or $2.5 \mathrm{pg}$ (Uchiyama et al., 2005). The tetraploid genome per nucleus having four copies adds up to $44 \mathrm{pg}$ that would be $22 \mathrm{pg}$ in early interphase having two copies. Apart from chromatin and non-histone proteins the nucleus consists of the nucleoplasm with sub-nuclear bodies that include the nucleoli, cajal bodies, PML bodies, speckles and nuclear membrane (Misteli \& Spector, 2011; Chu et al., 2017) whose mass should also be taken into account.

The size (area) of Pt stained and unstained (without Pt) nuclei from the ptychographic images showed variation indicating a different degree of spreading out on the membrane. Yet the range of masses seen is about the same for all the samples except two nuclei from sample 1 (Pt stained). This is supported by studies showing an increase of nuclear volume (besides increase in mass) in metaphase (Umen, 2005). Unfortunately, the two-dimensional projections do not directly relate to the volume of the nuclei. The mass should in principle be better correlated with the different stages of the cell cycle.

We suggest that the measurement method used in this study is sensitive enough to distinguish between different cell cycle states between which the DNA and histone in the nucleus differs by a factor of two. In the future a controlled study investigating nuclei at various cell cycle states (with synchronization at different stages) is needed with the described approach. The efficiency of the method could be significantly improved by refocusing the X-ray beam with reflective optics, and by employing on-the-fly scanning techniques to reduce motion control overhead (Clark et al., 2014). Overall, X-ray ptychography could serve useful for measuring nuclear masses in different cell types (normal and diseased) giving subcellular characteristics and assist in medical diagnosis as well as for biological research.

\section{Conclusion}

In this study, we investigated the application of ptychography for quantitative phase imaging of human cell nuclei. This approach is promising as the ptychographic reconstructions of the nuclei were much improved using the up-sampling technique, which is very successful when dealing with weakly scattering objects. The retrieved phase measurements of human nuclei show a wide distribution of nucleus mass that cannot be easily reconciled with theoretical estimates. The results show that the mass varies considerably. This can be explained by different aggregation phases of the nuclei, or variations in the binding of the $\mathrm{Pt}$ stain. A more robust study would both require a better understanding of the binding efficiency of the Pt stain and a larger sample size.

\section{Acknowledgements}

We acknowledge funding in the Research Complex at Harwell from a BBSRC Professorial Fellowship 'Diamond Professorial Fellowship for imaging chromosomes by coherent X-ray diffraction'. Work at Brookhaven National Laboratory was supported by the US Department of Energy, Office of Science, Office of Basic Energy Sciences, under Contract No. DESC0012704. Financial support was also provided by Professor El-Nasir Lalani at the Aga Khan University and generous donors.

\section{Funding information}

Funding is acknowledged from: Biotechnology and Biological Sciences Research Council (grant No. BB/H022597/1).

\section{References}

Alberts, B., Johnson, A., Lewis, J., Morgan, D., Raff, M., Roberts, K. \& Walter, P. (2014). Molecular Biology of the Cell, 6th ed. New York: Garland Science.

Bahr, G. F. \& Golomb, H. M. (1971). Proc. Natl Acad. Sci. USA, 68 , 726-730.

Batey, D. J., Edo, T. B., Rau, C., Wagner, U., Pešić, Z. D., Waigh, T. A. \& Rodenburg, J. M. (2014). Phys. Rev. A, 89, 043812.

Chu, F. Y., Haley, S. C. \& Zidovska, A. (2017). Proc. Natl Acad. Sci. 114, 10338-10343.

Clark, J. N., Huang, X., Harder, R. J. \& Robinson, I. K. (2014). Opt. Lett. 39, 6066-6069.

Dahl, K. N., Ribeiro, A. J. \& Lammerding, J. (2008). Circ. Res. 102, 1307-1318.

Dierolf, M., Menzel, A., Thibault, P., Schneider, P., Kewish, C. M., Wepf, R., Bunk, O. \& Pfeiffer, F. (2010a). Nature, 467, 436-439.

Dierolf, M., Thibault, P., Menzel, A., Kewish, C. M., Jefimovs, K., Schlichting, I., König, K., Bunk, O. \& Pfeiffer, F. (2010b). New J. Phys. 12, 035017.

Dunbar, D. \& Humphreys, G. (2006). ACM Trans. Graph. 25, 503508.

Edo, T. B., Batey, D. J., Maiden, A. M., Rau, C., Wagner, U., Pešić, Z. D., Waigh, T. A. \& Rodenburg, J. M. (2013). Phys. Rev. A, 87, 053850 .

Faulkner, H. M. L. \& Rodenburg, J. M. (2004). Phys. Rev. Lett. 93, 023903.

Giewekemeyer, K., Thibault, P., Kalbfleisch, S., Beerlink, A., Kewish, C. M., Dierolf, M., Pfeiffer, F. \& Salditt, T. (2010). Proc. Natl Acad. Sci. USA, 107, 529-534.

Golomb, H. M. \& Bahr, G. F. (1974). Chromosoma, 46, 233-245.

Heel, M. van \& Schatz, M. (2005). J. Struct. Biol. 151, 250-262.

Huang, X., Yan, H., Harder, R., Hwu, Y., Robinson, I. K. \& Chu, Y. S. (2014). Opt. Express, 22, 12634-12644.

Lamond, A. I. \& Earnshaw, W. C. (1998). Science, 280, 547-553. 
Maiden, A., Johnson, D. \& Li, P. (2017). Optica, 4, 736-745.

Maiden, A. M. \& Rodenburg, J. M. (2009). Ultramicroscopy, 109, 1256-1262.

Misteli, T. \& Spector, D. L. (2011). The Nucleus. New York: Cold Spring Harbor Laboratory Press.

Nishino, Y., Takahashi, Y., Imamoto, N., Ishikawa, T. \& Maeshima, K. (2009). Phys. Rev. Lett. 102, 018101.

Pederson, T. (2011). Cold Spring Harb. Perspect. Biol. 3, a000521.

Pešić, Z. D., Fanis, A. D., Wagner, U. \& Rau, C. (2013). J. Phys. Conf. Ser. 425, 182003.

Rau, C., Wagner, U., Pešić, Z. \& De Fanis, A. (2011). Phys. Status Solidi A, 208, 2522-2525.

Rippe, K. (2007). Curr. Opin. Genet. Dev. 17, 373-380.

Sandritter, W. \& Müller, D. (1959). Experientia, 15, 158-159.

Sandritter, W., Zilles, H. \& Kiefer, G. (1963). Acta Cytol. 7, 45-53.

Shemilt, L., Verbanis, E., Schwenke, J., Estandarte, A. K., Xiong, G., Harder, R., Parmar, N., Yusuf, M., Zhang, F. \& Robinson, I. K. (2015). Biophys. J. 108, 706-713.

Thibault, P., Dierolf, M., Menzel, A., Bunk, O., David, C. \& Pfeiffer, F. (2008). Science, 321, 379-382.

Thibault, P. \& Guizar-Sicairos, M. (2012). New J. Phys. 14, 063004.
Uchiyama, S., Kobayashi, S., Takata, H., Ishihara, T., Hori, N., Higashi, T., Hayashihara, K., Sone, T., Higo, D., Nirasawa, T., Takao, T., Matsunaga, S. \& Fukui, K. (2005). J. Biol. Chem. 280, 16994-17004.

Umen, J. G. (2005). Curr. Opin. Cell Biol. 17, 435-441.

Vries, R. I. de, Weijers, S., Bethke, K., Kogan, V., Vasterink, J., Kharchenko, A., Fransen, M. \& Bethke, J. (2007). Nucl. Instrum. Methods Phys. Res. A, 576, 164-168.

Wang, P., Bista, R., Bhargava, R., Brand, R. E. \& Liu, Y. (2010). Opt. Lett. 35, 2840-2842.

Wanner, G. \& Formanek, H. (1995). Chromosome Res. 3, 368-374.

Webster, M., Witkin, K. L. \& Cohen-Fix, O. (2009). J. Cell Sci. 122, 1477-1486.

Woodcock, C. L. F. \& Frank, J. (1984). J. Ultrastruct. Res. 89, 295-302. Yang, V. W. (2018). Physiology of the Gastrointestinal Tract, pp. $197-$ 219. Academic Press.

Yusuf, M., Chen, B., Hashimoto, T., Estandarte, A. K., Thompson, G. \& Robinson, I. (2014b). Biotechniques, 57, 302-307.

Yusuf, M., Parmar, N., Bhella, G. K. \& Robinson, I. K. (2014a). Biotechniques, 56, 257-261.

Zidovska, A. (2020). Biophys. Rev. 12, 1093-1106. 